\title{
Valutazione economica in base allo studio NAVoTRIAL01 con riferimento al contesto sanitario italiano: Vinorelbine orale e Cisplatino o Pemetrexed e Cisplatino seguiti da mantenimento rispettivamente con Vinorelbine orale e Pemetrexed nel trattamento del Carcinoma Polmonare Non a Piccole Cellule Non Squamoso (NS-NSCLC) in stadio avanzato
}

\author{
Francesco Grossi • Lucio Buffoni • Adolfo Favaretto • \\ Carlo Lucioni · Silvio Mazzi · Héctor Soto Parra \\ Pubblicato online: 5 marzo 2014 \\ (C) The Author(s) 2014. This article is published with open access at Springerlink.com
}

\begin{abstract}
Background Vinorelbine and Cisplatin is a standard treatment in non small cell lung cancer; oral Vinorelbine is registered in 45 countries. Pemetrexed and Cisplatin are recommended in front-line chemotherapy of nonsquamous non small cell lung cancer (NS-NSCLC). An economic evaluation of oral Vinorelbine plus Cisplatin and Pemetrexed plus Cisplatin was implemented in NS-NSCLC patients, adopting specific costs and clinical settings reflecting the Italian practice.

Materials and methods A cost evaluation was conducted from the perspective of the Italian National Health Service, based on a randomized phase II study in NS-NSCLC
\end{abstract}

F. Grossi ( $\square)$

U.O.S. Tumori Polmonari, IRCCS Azienda Ospedaliera

Universitaria San Martino IST, Istituto Nazionale per la Ricerca

sul Cancro, L.go R. Benzi, 10, 16132 Genova, Italia

e-mail: francesco.grossi@hsanmartino.it

F. Grossi

e-mail: fg1965@libero.it

L. Buffoni

Oncologia Medica 1, AO Città della Salute e della

Scienza-Ospedale Molinette, Torino, Italia

A. Favaretto

Oncologia Medica 2, Istituto Oncologico Veneto, IRCCS, Padova, Italia

C. Lucioni $\cdot$ S. Mazzi

Springer Healthcare Italia srl, Milano, Italia

H. Soto Parra

Oncologia Medica, P.O. Gaspare Rodolico, Azienda

Ospedaliero-Universitaria "Policlinico-Vittorio Emanuele",

Catania, Italia
(NAVoTRIAL01), with 100 oral Vinorelbine + Cisplatin patients (arm A) and 51 Pemetrexed + Cisplatin patients (arm B). Overall, Arm A/Arm B reported respectively: Disease Control Rate, including combination (4 cycles) and maintenance periods, of $75 \% / 76.5 \%$; median Progression Free and Overall Survival of 4.2/4.3 and 10.2/10.8 months.

Costs considered in the analysis were for anti-cancer drugs, administration settings (i.e. out-patient/in-patient/at home), serious adverse events (defined as involving hospitalization and suspected to be due to anti-cancer drugs), concomitant medications, blood transfusions.

Unit costs used for anti-cancer drugs were official exfactory prices, with further percent deductions enforced by law. The distribution of administration settings was remodelled according to the respective frequencies found for the subset of Italian patients participating in NAVoTRIAL01; for out/in-patient settings, DRG (Diagnosis-Related Group) and other tariffs (day-hospital or one day admission) were used (no cost was charged when administration was at home). Hospitalization costs were assessed for serious adverse events on the basis of appropriate DRG tariffs. For concomitant medications, prices were ex-factory or $50 \%$ of retail price. Blood transfusions were evaluated using public tariffs.

Results The average cost per patient for the overall treatment was $€ 4,511$ (of which: $€ 1,763$ for anti-cancer drugs, $€ 1,801$ for administration settings, and $€ 611$ for serious adverse events) in arm A; the respective costs were $€ 14,793$ $(€ 13,615, € 344, € 567)$ in arm B.

Conclusions Given the reported efficacy outcomes with both regimens, oral Vinorelbine + Cisplatin followed by maintenance with oral Vinorelbine provides substantial savings ( $€ 10,282$ per patient on average), appearing a cost-effective 
treatment option in advanced non-squamous non small cell lung cancer. Sensitivity analysis shows the robustness of the results, which should be confirmed by a phase III trial.

\section{Introduzione}

In Italia il tumore del polmone rappresenta la prima causa di morte per tumore nella popolazione maschile e la terza in quella femminile [1].

La terapia standard per il trattamento della prima linea del Carcinoma Polmonare Non a Piccole Cellule (NSCLC) è tuttora rappresentata da doppiette con Cisplatino o Carboplatino e farmaci di terza generazione quali Vinorelbina, Gemcitabina, Paclitaxel o Docetaxel somministrate per 4-6 cicli [2-4].

Negli ultimi anni alcuni studi hanno suggerito l'istologia come fattore discriminante per la scelta terapeutica. Un'analisi di sottogruppo di uno studio prospettico randomizzato di non inferiorità con Cisplatino + Pemetrexed verso Cisplatino + Gemcitabina ha mostrato un vantaggio in sopravvivenza mediana per la combinazione con Pemetrexed negli istotipi non squamosi rispetto al braccio di confronto [5]. Pemetrexed è al momento registrato e rimborsato in Italia in associazione a Cisplatino per il trattamento di prima linea in pazienti affetti da NSCLC localmente avanzato o metastatico con istotipo non squamoso. Le linee guida dell'European Society of Medical Oncology (ESMO) [3] riportano che l'utilizzo di Pemetrexed e Cisplatino è da preferire a Gemcitabina e Cisplatino in pazienti con NSCLC non squamoso (NS-NSCLC) con un livello di evidenza II e un grado di raccomandazione B. In letteratura, non sono presenti studi prospettici di fase III che abbiano confrontato Pemetrexed + Cisplatino con altri regimi a base di platino in pazienti ad istologia non squamosa. I risultati dello studio NAVoTRIAL01, base clinica di questa analisi, meriterebbero una conferma in uno studio più ampio di fase III. In associazione a Carboplatino, Pemetrexed, in pazienti con NS-NSCLC, è stato invece confrontato a Docetaxel e Carboplatino [6]. In questo studio la sopravvivenza mediana, pur essendo un end-point secondario, non era differente da quella del regime di confronto. Più recentemente Pemetrexed ha fallito nel dimostrare un vantaggio in sopravvivenza mediana quando associato a Bevacizumab e Carboplatino seguito da mantenimento con Pemetrexed + Bevacizumab verso Bevacizumab + Carboplatino + Paclitaxel seguito da mantenimento con Bevacizumab in pazienti con NS-NSCLC [7].

Vinorelbine, in forma orale o endovenosa, si è dimostrata efficace in numerosi studi sia negli stadi avanzati [8-15] che nella terapia adiuvante $[16,17]$ del NSCLC, inoltre la formulazione orale offre diversi vantaggi dal punto di vista gestionale ed è preferita dal paziente [13, 18]. Vinorelbine orale è registrata e rimborsata in Italia con indicazione carcinoma polmonare non a piccole cellule.
Alcune esperienze in cui è stata utilizzata Vinorelbine, $[15,16,19]$ mostrano, in analisi per sottogruppi, come l'istotipo adenocarcinoma sembri essere caratterizzato da una maggiore responsività agli schemi chemioterapici di ultima generazione.

Infine, la terapia di mantenimento, per pazienti con NSCLC non in progressione dopo una fase d'induzione con 4 cicli di chemioterapia a base di platino, è un settore su cui la ricerca si è focalizzata in questi ultimi anni [20-24]. In linea con le evidenze al momento disponibili, la terapia di mantenimento può essere un'opzione da condividere con il paziente $[2,4]$.

Date queste premesse, lo studio NAVoTRIAL01 [25] ha valutato, in pazienti con NS-NSCLC, due schemi di trattamento a base di Cisplatino (associato a Vinorelbine orale o Pemetrexed) seguiti da continuation maintenance con i rispettivi agenti singoli Vinorelbine orale e Pemetrexed. Entrambi i trattamenti hanno mostrato efficacia in termini di controllo di malattia (obiettivo primario) e sopravvivenza mediana e libera da progressione. Avendo i due regimi chemioterapici dei costi differenti, si è condotta un' analisi economica basata sui risultati di questo studio per valutare se il trattamento con Vinorelbine orale consentisse un risparmio di risorse nella prospettiva economica del Sistema Sanitario Nazionale italiano (SSN).

\section{Materiali e metodi}

\subsection{La base clinica}

Lo studio clinico di riferimento per la presente analisi economica è il NAVoTRIAL01 [25]. Si tratta di uno studio prospettico randomizzato (2:1) di fase II, condotto presso 34 centri in 11 Paesi tra il 2009 e il 2011, su pazienti con NSNSCLC in stadio avanzato. L'obiettivo primario era di valutare, nella popolazione Intention To Treat (ITT), l'efficacia in termini di controllo della malattia $(\mathrm{DCR}=$ risposte complete + risposte parziali + stabilità di malattia valutate secondo criteri RECIST 1.1 [26]) di due regimi antitumorali.

Nel braccio $\mathrm{A}, 100$ pazienti (età media 62 anni, $62 \%$ maschi) sono stati trattati con Vinorelbine orale + Cisplatino. Durante il periodo di induzione (4 cicli ogni 21 giorni), nel giorno 1 del ciclo sono stati somministrati Vinorelbine orale $\left(60 \mathrm{mg} / \mathrm{m}^{2}\right.$ nel primo ciclo, poi, in assenza di tossicità rilevanti, $80 \mathrm{mg} / \mathrm{m}^{2}$ nei cicli successivi) e Cisplatino (80 $\mathrm{mg} / \mathrm{m}^{2}$ ); nel giorno 8 era somministrata solo Vinorelbine orale con lo stesso dosaggio del rispettivo giorno 1. I pazienti che ottenevano una risposta o stabilità di malattia hanno iniziato un successivo periodo di mantenimento con sola Vinorelbine orale (al dosaggio del ciclo 4) nei giorni 1 e 8 ogni 3 settimane fino a progressione. Nel braccio B sono stati trattati 51 pazienti (età media 63,8 anni, $65 \%$ maschi). Nei 
quattro cicli (di 21 giorni) del periodo di induzione Pemetrexed e Cisplatino sono stati somministrati in prima giornata rispettivamente a $500 \mathrm{mg} / \mathrm{m}^{2}$ e $75 \mathrm{mg} / \mathrm{m}^{2}$. I pazienti che presentavano risposta obiettiva o stabilità di malattia hanno iniziato un successivo periodo di mantenimento con solo Pemetrexed somministrato ogni 21 giorni fino a progressione, con lo stesso dosaggio del ciclo 4 .

In base al disegno statistico dello studio, entrambi i trattamenti sono risultati efficaci: il tasso di controllo della malattia è stato, considerando la popolazione ITT, del $75 \%$ nel braccio A (I.C. 95\% 65,3-83,1) e del 76,5\% nel braccio B (I.C. $95 \% 62,5-87,2$ ). I rispettivi profili di tolleranza sono risultati differenti ma facilmente gestibili.

Tra gli obiettivi secondari, la sopravvivenza mediana libera da progressione è stata di 4,2 (I.C. $95 \%$ 3,6-4,7) e 4,3 (I.C. 95\% 3,8-5,6) mesi rispettivamente per il braccio A e $\mathrm{B}$ ed il tempo mediano di sopravvivenza complessiva è stato di 10,2 (I.C. 95\% 7,8-11,9) e 10,8 (I.C. 95\% 7,0-16,4) mesi rispettivamente nel braccio A e B. Questi dati fanno riferimento a tutta la popolazione in studio, compresi i pazienti che andavano in progressione durante la fase di induzione.

\subsection{Il metodo di analisi}

In generale, l'obiettivo di una valutazione farmacoeconomica è fare dei confronti fra due o più trattamenti, sulla base dei rispettivi costi e risultati, al fine di compiere una scelta razionale, dal punto di vista del terzo pagante, fra le alternative [27]. Considerato l'outcome primario dello studio di riferimento sopra presentato (efficacia di entrambi i trattamenti in termini di DCR), il confronto alla base del presente lavoro si rivolge alla valutazione dei costi dei due trattamenti in base a quanto osservato nello studio, individuando nel trattamento meno costoso un possibile criterio di preferibilità. La metodologia qui adottata è dunque quella nota come analisi dei costi, nella prospettiva del SSN.

Per tutte le elaborazioni è stato utilizzato un database popolato con i dati di utilizzo delle risorse a livello di paziente riportati nei dataset del NAVoTRIAL01.

Per ogni braccio di trattamento sono stati presi in considerazione i seguenti parametri: numero totale di somministrazioni dei chemioterapici; dose media somministrata (media aritmetica); frequenza di utilizzo dei diversi settings (tipologie) di somministrazione (out-patient: accesso non superiore alle 12 ore; in-patient: ricovero ordinario; at home: assunzione del farmaco a domicilio); ospedalizzazioni per eventi avversi (Serious Adverse Events, SAE) la cui correlazione con i farmaci in studio fosse definita almeno "possibile" dagli sperimentatori; trattamenti concomitanti utilizzati in seguito all'insorgenza di eventi avversi in corso di chemioterapia; trasfusioni al di fuori di eventuali fatte in ambito di SAE.
2.3 I costi unitari delle risorse

\subsubsection{Farmaci antitumorali}

Il costo dei farmaci antitumorali è stato calcolato (Cap. 3.1) in base al numero di somministrazioni e di dosaggi medi utilizzati per ogni singolo farmaco per ciascun periodo di trattamento (induzione e mantenimento) e in funzione della tipologia di rimborso associata alla somministrazione.

\section{Costi unitari dei farmaci antitumorali Vedi Tabella 1.}

Criteri di attribuzione del costo dei farmaci antitumorali al SSN

\section{Vinorelbine orale}

- nel giorno 1, durante il periodo d'induzione, il costo è "zero" (non rappresenta un costo aggiuntivo per il SSN) in quanto compreso nel DRG di somministrazione del Cisplatino [30, 31].

- nel giorno 8 del periodo di induzione e in entrambi i giorni nel periodo di mantenimento, avendo qui adottato la regolamentazione MAC $04,{ }^{1}$ il costo è rimborsato con procedura di file $\mathrm{F}$ (rappresenta quindi un costo addizionale a carico del SSN); è rimborsato anche se il setting è at-home [32].

Pemetrexed Il costo è rimborsato con procedura di File F (rappresenta quindi un costo addizionale a carico del SSN) $[32,33]$.

Cisplatino Quando somministrato con Vinorelbine orale il costo è "zero" in quanto compreso nel DRG di somministrazione [30, 31]. Quando somministrato con Pemetrexed, avendo qui adottato la regolamentazione MAC $01,{ }^{1}$ il costo viene rimborsato con procedura di File $\mathrm{F}$ [32].

\subsubsection{Settings di somministrazione}

Per la valorizzazione dei diversi settings di somministrazione (cui corrisponde una determinata tipologia di prestazione) è stata utilizzata la tariffazione riportata in Tabella 2.

Ai fini di una migliore trasferibilità dello studio alla pratica italiana, la distribuzione (ovvero la frequenza di utilizzo) dei diversi settings di somministrazione (out-patient, inpatient, at home) rilevata nel NAVoTRIAL01 è stata ricondotta a quella dei soli pazienti italiani partecipanti al trial stesso. ${ }^{2} \mathrm{Si}$ è inoltre convenuto che, nei rari casi in cui il setting non fosse indicato, la relativa frequenza fosse attribuita all'out-patient (il setting più utilizzato).

\footnotetext{
${ }^{1} \mathrm{Si}$ veda il seguente Par. 2.3.2.

${ }^{2} \mathrm{Si}$ tratta di 22 pazienti (13 nel braccio A e 9 nel braccio B). Le frequenze di utilizzo sono state stimate sulla base del numero delle somministrazioni fruite dagli stessi nei diversi settings.
} 
Tabella 1 Costo unitario dei farmaci antitumorali

\begin{tabular}{llll}
\hline Principio attivo & Nome commerciale & $€ /$ confezione & $€ / \mathrm{mg}$ \\
\hline Vinorelbine bitartrato & Navelbine 1 cps 30 mg & 103,04 (prezzo al pubblico) [28] & 1,56 al netto degli sconti di legge \\
Pemetrexed & Alimta ev 1 fl 500 mg & $\begin{array}{l}1.111,93 \text { (prezzo massimo di } \\
\text { cessione al SSN) [29] }\end{array}$ & 2,22 \\
Cisplatino & Generici & $\begin{array}{l}\text { Prezzi all'ospedale di 13 confezioni } \\
\text { di generici }\end{array}$ & $\begin{array}{l}0,35 \text { [28] prezzi riportati al mg e aggregati } \\
\text { con media aritmetica }\end{array}$ \\
\hline
\end{tabular}

Tabella 2 Valorizzazione dei settings di somministrazione

\begin{tabular}{|c|c|c|c|}
\hline & Out-patient & In-patient & At home \\
\hline \multicolumn{4}{|l|}{ Braccio A } \\
\hline \multicolumn{4}{|l|}{ Induzione } \\
\hline Giorno 1 & $\begin{array}{l}\text { DRG 410a } \\
\text { Tariffa DH: } € 431[31]\end{array}$ & $\begin{array}{l}\text { DRG } 410^{\mathrm{a}} \\
\text { Tariffa } 1 \text { giorno: } € 359 \text { [31] }\end{array}$ & Setting non utilizzato \\
\hline Giorno 8 & $\begin{array}{l}\text { Pacchetto MAC } 04^{\mathrm{b}} \\
\text { Tariffa: } € 44[32]\end{array}$ & Setting non utilizzato & Costo 0 \\
\hline Mantenimento & $\begin{array}{l}\text { Pacchetto MAC } 04^{\mathrm{b}} \\
\text { Tariffa: } € 44[32]\end{array}$ & Setting non utilizzato & Setting non utilizzato \\
\hline \multicolumn{4}{|l|}{ Braccio B } \\
\hline Induzione & $\begin{array}{l}\text { Pacchetto MAC } 01^{\mathrm{c}} \\
\text { Tariffa: } € 44[32]\end{array}$ & $\begin{array}{l}\text { DRG } 410^{a} \\
\text { Tariffa } 1 \text { giorno: } € 359 \text { [31] }\end{array}$ & Setting non utilizzato \\
\hline Mantenimento & $\begin{array}{l}\text { Pacchetto MAC } 01^{\mathrm{c}} \\
\text { Tariffa: } € 44[32]\end{array}$ & Setting non utilizzato & \\
\hline
\end{tabular}

Braccio A: Vinorelbine orale (VNRo) + Cisplatino (CDDP) $\rightarrow$ VNRo; Braccio B: Pemetrexed (PEM) + CDDP $\rightarrow$ PEM

${ }^{a}$ DRG 410: Chemioterapia non associata a diagnosi secondaria di leucemia acuta

${ }^{b}$ MAC 04: Terapia antitumorale con somministrazione di farmaci orali o IM

${ }^{\mathrm{c}}$ MAC 01: Chemioterapia con somministrazione di farmaci ad alto costo

Per l'applicazione dei DRG alla valorizzazione delle risorse è stata di regola adottata la Tariffa Unica Convenzionale (TUC) [31], la sola ad avere validità su tutto il territorio nazionale essendo alla base delle compensazioni interregionali.

Nei casi indicati in Tabella 2 si è fatto ricorso alle tariffe di Macroattività Ambulatoriale Complessa (MAC) [32], ciò nell'ipotesi che, pur trattandosi della regolamentazione adottata dalla Lombardia, essa fosse probabilmente utilizzata con valori similari anche in altre realtà regionali. Questo aspetto è stato oggetto di un'analisi di sensibilità i cui risultati sono riportati nel Par. 3.7.3.

\subsubsection{Eventi avversi}

Sono stati considerati nell'analisi solo gli eventi rilevanti dal punto di vista sia clinico che economico, cioè definiti come quelli soggetti a ospedalizzazione e in sospetto (valutazione degli sperimentatori) di essere causati dai farmaci in studio. Nel braccio A i SAE rilevanti sono stati 18 (su un totale di 134 eventi avversi), mentre nel braccio B sono stati 8 (su 71).
Su indicazioni raccolte da pareri esperti, per valorizzare tali eventi sono stati utilizzati i seguenti DRG (Tabella 3), intendendo che la relativa tariffa fosse comprensiva di tutte le prestazioni, farmaci in particolare, richieste per il trattamento dell'evento [31].

\subsubsection{Trattamenti farmacologici concomitanti}

Nel braccio A si sono registrate 3.066 prescrizioni di trattamento (su tutti i 100 pazienti partecipanti), mentre 1.966 sono state registrate nel braccio B (su tutti i 51 pazienti). L'insieme delle prescrizioni è stato qui suddiviso in due gruppi, uno denominato "preventivo" (pari al 52\% nel braccio A e al 68\% nel braccio B), comprendente i farmaci somministrati in occasione della chemioterapia e uno "curativo", comprendente i farmaci somministrati in momenti diversi dal trattamento chemioterapico.

Al fine della stima dei costi, i trattamenti preventivi non sono stati considerati, nell'ipotesi che il loro costo fosse compreso nelle tariffe adottate per la somministrazione della chemioterapia. Infatti la maggior parte di essi (il 55\% nel braccio A e il $52 \%$ nel braccio B) è costituita da farmaci antiemetici. 
Le prescrizioni comprese nel gruppo curativo ( $48 \%$ del totale nel braccio A e $32 \%$ nel braccio B) erano distribuite su un gran numero di voci farmacologiche (465 e 266 rispettivamente nei due bracci), spesso diverse tra loro solo per variazioni nella denominazione usata. A fronte di questa complessità (legata sia al numero che alla variabilità delle denominazioni utilizzate), nella presente analisi sono state fatte delle restrizioni e delle approssimazioni, limitandosi a mantenere in evidenza le informazioni più significative. L'analisi ha evidenziato 478 prescrizioni nel braccio A (quasi 5 prescrizioni in media per paziente) e 224 prescrizioni nel braccio $\mathrm{B}$ (poco più di 4 per paziente). Esse sono state raggrup-

Tabella 3 Valorizzazione degli eventi avversi che hanno richiesto ospedalizzazione

\begin{tabular}{lll}
\hline Evento avverso (da solo o principale) & DRG & Tariffa (€) [31] \\
\hline Embolia polmonare & $078^{\mathrm{a}}$ & 3.804 \\
Polmonite & $089^{\mathrm{b}}$ & 3.307 \\
Vomito & $183^{\mathrm{c}}$ & 1.170 \\
Iponatremia & $297^{\mathrm{d}}$ & 2.265 \\
Insufficienza renale & $316^{\mathrm{e}}$ & 3.511 \\
Anemia & $395^{\mathrm{f}}$ & 2.071 \\
Affaticamento/dolore gastrico & $464^{\mathrm{g}}$ & 1.759 \\
Neutropenia febbrile & $574^{\mathrm{h}}$ & 5.138 \\
\hline
\end{tabular}

a'DRG 078: Embolia polmonare

${ }^{\mathrm{b}}$ DRG 089: Polmonite, età maggiore 17 anni, con cc

${ }^{c}$ DRG 183: Esofagite, gastroenterite e miscellanea di malattie dell'apparato digerente, età $>17$ anni, senza cc

${ }^{\mathrm{d}}$ DRG 297: Disturbi della nutrizione e miscellanea di disturbi del metabolismo, età $>17$ anni, senza cc

${ }^{e}$ DRG 316: Insufficienza renale

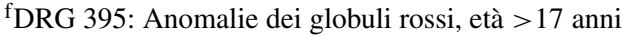

${ }^{\mathrm{g}}$ DRG 464: Segni e sintomi senza cc

${ }^{\mathrm{h}}$ DRG 574: Diagnosi ematologiche/immunologiche maggiori, eccetto anemia falciforme e coagulopatie pate per categorie (ad esempio "antimicotici") all'interno di ciascuna delle quali-per semplificare le elaborazioniè stato individuato il principio attivo prevalente considerato, in prima approssimazione, rappresentativo di tutta la categoria. Al fine della valorizzazione del costo delle prescrizioni, tra i prodotti con cui il principio attivo prescelto è presente sul mercato si è poi selezionato quello più economico (ad es. il generico, quando esistente) e la confezione più economica. Per ciascuna prescrizione si è infine calcolato un costo (Tabella 4) sulla base del prezzo (ex-factory o al pubblico scontato del 50\%) e delle indicazioni di dosaggio e durata del trattamento desunte dal Riassunto delle Caratteristiche del Prodotto [28].

\subsubsection{Trasfusioni}

Sono state considerate soltanto quelle effettuate extra ricovero, in quanto il costo delle altre è già compreso nella tariffa del DRG assegnato al ricovero in cui sono state effettuate. Al costo dell'emoderivato è stato aggiunto quello della prestazione ambulatoriale per la procedura di trasfusione (Tabella 5).

\subsection{Analisi di sensibilità}

Con un'analisi di tipo deterministico (a una via) è stata valutata la tenuta dei risultati al variare di alcune condizioniquadro (tra le quali non la distribuzione dei setting di somministrazione, essendosi adottata di proposito quella dei pazienti italiani nel NAVoTRIAL01 per simulare la realtà italiana).

Essendo il costo totale dei farmaci antitumorali anche correlato ai dosaggi, i valori medi delle dosi sono stati portati, in via esplorativa, agli estremi dei rispettivi intervalli di confidenza.

Inoltre, sono stati fatti variare i prezzi di Vinorelbine orale e Pemetrexed per individuare un ipotetico punto di pareggio tra i costi complessivi dei due trattamenti.

Tabella 4 Valorizzazione dei trattamenti concomitanti "curativi"

\begin{tabular}{|c|c|c|c|c|c|c|}
\hline $\begin{array}{l}\text { Trattamenti concomitanti per } \\
\text { categoria farmacologica }\end{array}$ & $\begin{array}{l}\text { Principio attivo } \\
\text { prevalente }\end{array}$ & $\begin{array}{l}\mathrm{N}^{\circ} \text { prescrizioni } \\
\text { braccio A }\end{array}$ & $\begin{array}{l}\mathrm{N}^{\circ} \text { prescrizioni } \\
\text { braccio } \mathrm{B}\end{array}$ & Dose/die & $\begin{array}{l}\text { Durata } \\
(\mathrm{gg})\end{array}$ & $\begin{array}{l}\text { Costo per } \\
1 \text { trattamento }(€)\end{array}$ \\
\hline Antimicotici & Fluconazolo & 12 & 10 & $400 \mathrm{mg}$ & 7 & 40,2 \\
\hline Antibiotici & Amoxicillina & 29 & 26 & $3 \mathrm{~g}$ & 4 & 4,21 \\
\hline Antidiarroici & Loperamide & 13 & 5 & $4 \mathrm{mg}$ & 4 & 0,56 \\
\hline Antiemetici 5HT3 antagonisti & Ondansetron & 64 & 21 & $16 \mathrm{mg}$ & 5 & 114,8 \\
\hline Antiemetici non 5HT3 antagonisti & Metoclopramide & 72 & 32 & $30 \mathrm{mg}$ & 4 & 1,75 \\
\hline Stimolanti l'eritropoiesi (ESA) & Eritropoietina & 12 & 7 & $10^{4} \mathrm{UI}$ & 12 & 1.022 \\
\hline Fattori di crescita (G-CSF) & Filgrastim & 13 & 2 & $350 \mathrm{mg}$ & 7 & 491 \\
\hline Analgesici non oppioidi & Paracetamolo & 159 & 67 & $2 \mathrm{~g}$ & 3 & 2,07 \\
\hline Analgesici oppioidi & Tramadolo & 104 & 54 & $300 \mathrm{mg}$ & 3 & 2,32 \\
\hline
\end{tabular}

Braccio A: VNRo + CDDP $\rightarrow$ VNRo; Braccio B: PEM + CDDP $\rightarrow$ PEM 
Infine, considerando la variabilità esistente tra le Regioni italiane sui meccanismi di rimborsabilità per somministrazione di chemioterapia, è stato intrapreso un ampio confronto interregionale per valutare l'estensione di tale variabilità e, implicitamente, l'impatto sui risultati di base che sono riconducibili, per molti aspetti, alla normativa vigente in Lombardia.

\section{Risultati}

I risultati sono espressi come costi medi per paziente ottenuti dividendo, per ciascun braccio, l'ammontare complessivo del costo lungo tutto il trattamento per il numero dei pazienti randomizzati.

\subsection{Il costo dei farmaci antitumorali}

Per un'esposizione più dettagliata dei risultati e per evidenziare i diversi criteri di attribuzione del costo dei farmaci antitumorali (Tabella 6, colonna costo/mg attibuito); sono state tenute separate le due fasi di trattamento ed il giorno 1 e 8 del periodo d'induzione.

Non sono state prese in considerazione le somministrazioni registrate con dose zero in quanto di fatto non effettua- te. Si tratta: per Cisplatino di 5 somministrazioni (2 pazienti) nel braccio con Vinorelbine orale e di una nel braccio con Pemetrexed tutte per funzionalità renale non ottimale; per Vinorelbine orale di 41 somministrazioni il giorno 8 del periodo di induzione, di cui solo 20 per motivazioni correlate al farmaco, e di 9 il giorno 8 del periodo di mantenimento di cui solo 2 dovute al farmaco.

Il costo di Vinorelbine orale nel periodo di mantenimento è circa doppio di quello nel periodo d'induzione perché, in questo periodo, al giorno 1 il costo dei farmaci chemioterapici è compreso nella tariffa del DRG 410, così come lo è il costo di cisplatino nello stesso braccio A.

Nel braccio B, invece, c'è equivalenza di costo tra $\mathrm{i}$ due suddetti periodi perché sia Pemetrexed che Cisplatino sono sempre rimborsati all'ospedale, con procedura di File F.

Il costo complessivo medio per paziente di Pemetrexed (€13.615) si evidenzia ampiamente multiplo dell' analogo di Vinorelbine orale (€1.763).

\subsection{Il costo dei settings di somministrazione}

Per il calcolo del costo dei settings di somministrazione, corrispondendo per ciascuna tipologia una diversa tariffa di rimborso, è stato importante rispecchiare il più possibile la pratica italiana. Come si osserva in Tabella 7, confrontando le frequenze di accesso alle tre tipologie di somministrazione tra la popolazione totale dello studio e quella dei soli pazienti italiani, emerge un differente utilizzo delle risorse. Di

Tabella 5 Valorizzazione degli emoderivati

\begin{tabular}{lc}
\hline Tipologia della risorsa & Tariffa (€) \\
\hline Concentrato eritrocitario $(280 \mathrm{ml} \pm 20 \%)$ & $153[34]$ \\
Concentrato piastrinico da pool di buffy coat $\left(2,5 * 10^{11}\right.$ piastrine $)$ & $115[34]$ \\
Procedura di trasfusione di sangue o emocomponenti & $25,8[35]$ \\
\hline
\end{tabular}

Tabella 6 Costo dei farmaci antitumorali

\begin{tabular}{|c|c|c|c|c|c|c|c|c|c|c|}
\hline \multirow[t]{2}{*}{ Periodo di somministrazione } & \multicolumn{5}{|c|}{ Braccio A $(n=100)$} & \multicolumn{5}{|c|}{ Braccio $\mathrm{B}(n=51)$} \\
\hline & $\begin{array}{l}\mathrm{N}^{\circ} \\
\text { sommini- } \\
\text { strazioni }\end{array}$ & $\begin{array}{l}\text { Dose } \\
\text { media } \\
(\mathrm{mg})\end{array}$ & $\begin{array}{l}\text { Costo/mg } \\
\text { attribuito } \\
(€)\end{array}$ & $\begin{array}{l}\text { Costo/ } \\
\text { dose } \\
(€)\end{array}$ & $\begin{array}{l}\text { Costo } \\
\text { complessivo } \\
(€)\end{array}$ & $\begin{array}{l}\mathrm{N}^{\circ} \\
\text { sommini- } \\
\text { strazioni }\end{array}$ & $\begin{array}{l}\text { Dose } \\
\text { media } \\
(\mathrm{mg})\end{array}$ & $\begin{array}{l}\text { Costo/mg } \\
\text { attribuito } \\
(€)\end{array}$ & $\begin{array}{l}\text { Costo/ } \\
\text { dose } \\
(€)\end{array}$ & $\begin{array}{l}\text { Costo } \\
\text { complessivo } \\
(€)\end{array}$ \\
\hline & VNRo & & & & & PEM & & & & \\
\hline \multicolumn{11}{|l|}{ Induzione } \\
\hline Giorno 1 & 333 & 126,2 & 0 & 0,0 & 0 & 176 & 859,5 & 2,22 & 1908,1 & 335.824 \\
\hline Giorno 8 & 291 & 126,1 & 1,56 & 196,7 & 57.244 & - & - & - & - & - \\
\hline Totale & 624 & & & & 57.244 & 176 & & & & 335.824 \\
\hline \multirow[t]{2}{*}{ Mantenimento } & 567 & 134,6 & 1,56 & 210,0 & 119.056 & 181 & 872,8 & 2,22 & 1937,6 & 350.708 \\
\hline & CDDP & & & & & CDDP & & & & \\
\hline Induzione & 328 & 136,7 & 0 & 0,0 & $\mathbf{0}$ & 175 & 127,3 & 0,35 & 44,6 & 7.797 \\
\hline Totale trattamento & & & & & 176.301 & & & & & 694.329 \\
\hline $\mathrm{N}^{\circ}$ pazienti & & & & & 100 & & & & & 51 \\
\hline Costo medio per paziente & & & & & 1.763 & & & & & 13.615 \\
\hline
\end{tabular}




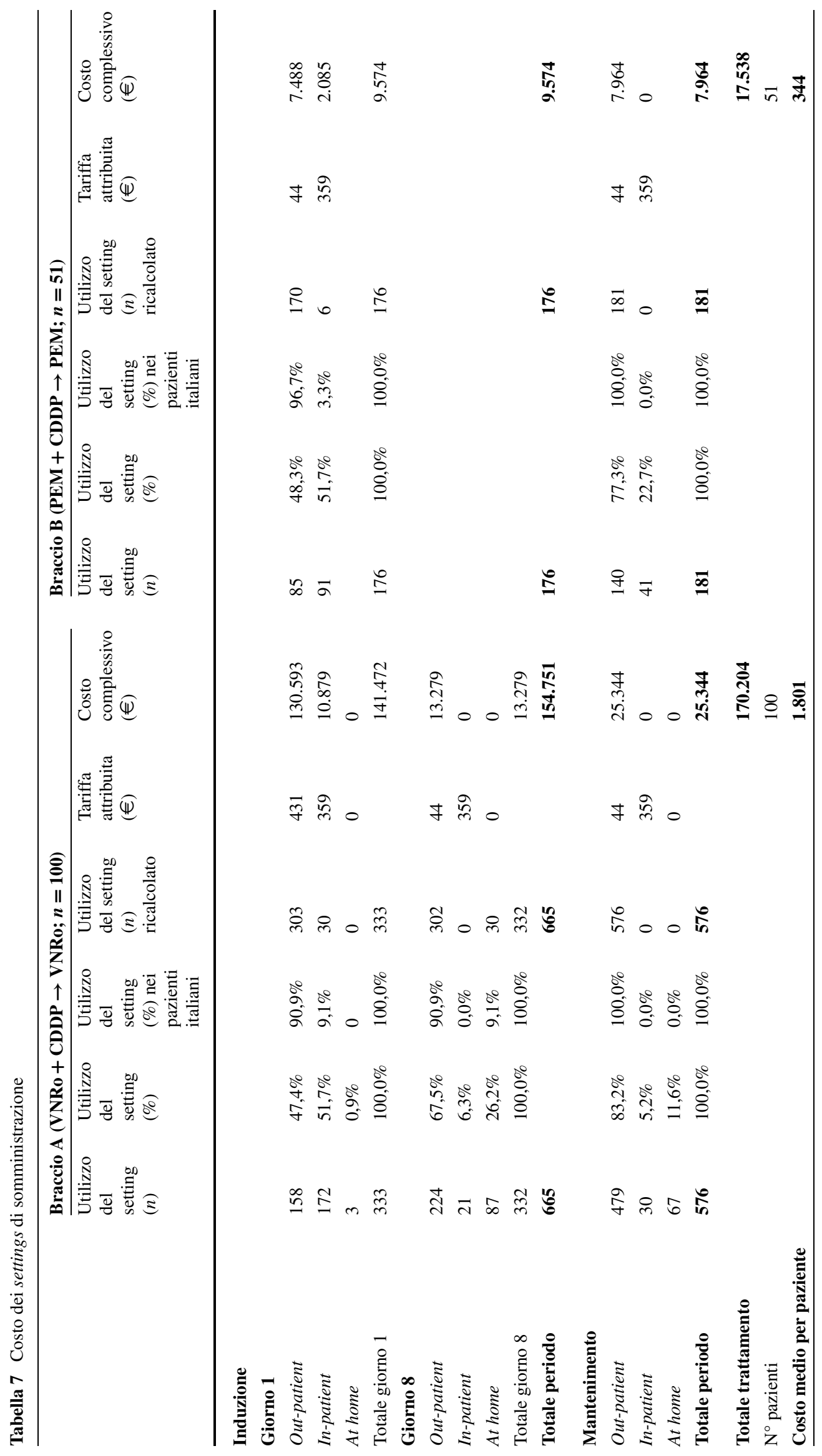


Tabella 8 Costo degli eventi avversi con ospedalizzazione

\begin{tabular}{|c|c|c|c|c|c|c|}
\hline & \multirow[b]{2}{*}{$\begin{array}{l}\text { DRG } \\
\text { applicato }\end{array}$} & \multirow[b]{2}{*}{ Tariffa } & \multicolumn{2}{|c|}{$\begin{array}{l}\text { Braccio A } \\
\text { VNRo }+ \text { CDDP } \rightarrow \text { VNRo; } n=100\end{array}$} & \multicolumn{2}{|c|}{$\begin{array}{l}\text { Braccio B } \\
\text { PEM + CDDP } \rightarrow \text { PEM; } n=51\end{array}$} \\
\hline & & & $\begin{array}{l}\text { Frequenza } \\
\text { dell'evento }\end{array}$ & $\begin{array}{l}\text { Costo } \\
\text { complessivo (€) }\end{array}$ & $\begin{array}{l}\text { Frequenza } \\
\text { dell'evento }\end{array}$ & $\begin{array}{l}\text { Costo } \\
\text { complessivo (€) }\end{array}$ \\
\hline \multicolumn{7}{|l|}{ Ematologici } \\
\hline Neutropenia febbrile & 574 & 5.138 & 8 & 41.104 & 3 & 15.414 \\
\hline Anemia & 395 & 2.071 & 2 & 4.142 & 2 & 4.142 \\
\hline Totale & & & 10 & 45.246 & 5 & 19.556 \\
\hline \multicolumn{7}{|l|}{ Non ematologici } \\
\hline Vomito & 183 & 1.170 & 3 & 3.510 & & \\
\hline Insufficienza renale & 316 & 3.511 & 2 & 7.022 & & \\
\hline Affaticamento/dolore gastrico & 464 & 1.759 & 3 & 5.277 & & \\
\hline Embolia polmonare & 078 & 3.804 & & & 1 & 3.804 \\
\hline Polmonite & 089 & 3.307 & & & 1 & 3.307 \\
\hline Iponatremia & 297 & 2.265 & & & 1 & 2.265 \\
\hline Totale & & & 8 & 15.809 & 3 & 9.376 \\
\hline Totale eventi avversi & & & 18 & 61.055 & 8 & 28.932 \\
\hline $\mathrm{N}^{\circ}$ pazienti & & & & 100 & & 51 \\
\hline Costo medio per paziente & & & & 611 & & 567 \\
\hline
\end{tabular}

conseguenza, il calcolo dei costi è stato effettuato riproporzionando il totale degli accessi alle frequenze di utilizzo rilevate nel campione di pazienti italiani per giornata e periodo di somministrazione.

$\mathrm{Nel}$ caso di somministrazioni riportate nel database con dose 0 in quanto non effettuate (cfr. Cap. 3.1), era comunque registrato, nello studio, l'utilizzo di un setting ed è quindi stato conteggiato.

La giustificazione delle tariffe attribuite è reperibile nella Tabella 2.

Nel braccio A, il costo del setting di somministrazione nel periodo d'induzione risulta, pur tenendo conto della diversa numerosità di pazienti, più elevato dell'analogo nel braccio B perché quando al giorno 1 la somministrazione è in out-patient (cioè più o meno nel $90 \%$ dei casi in entrambi i bracci) si applicano due diverse tariffe: $€ 431$ (DRG 410) nel braccio A e $€ 44$ (MAC01) nel braccio B (cfr. Tabella 2).

La distinzione tra il giorno 1 e il giorno 8 nel periodo di induzione esplicita inoltre, nel braccio A, l'impiego di differenti tariffe di valorizzazione dell'out-patient (€431 e 44 rispettivamente nei due giorni).

Il costo medio di somministrazione per paziente dell'intero trattamento nel braccio A $(€ 1.801)$ risulta alla fine il quintuplo di quello nel braccio B (€344).

\subsection{Il costo degli eventi avversi}

La tipologia degli eventi avversi di natura ematologica riportati (neutropenia febbrile e anemia) è comune a entrambi i bracci, mentre appare ben differenziata negli eventi non ematologici (Tabella 8). Nel complesso, comunque, l'incidenza degli eventi avversi risulta relativamente bilanciata sia nella frequenza (18 eventi nel braccio $\mathrm{A}$, cioè 1,8 eventi per mille pazienti; 8 nel braccio $B$, cioè 1,6 per mille) che nel costo medio per paziente ( $€ 611$ nel braccio A e $€ 567$ nel braccio $\mathrm{B})$.

\subsection{Il costo dei trattamenti farmacologici concomitanti}

Pur con la variabilità di peso delle rispettive frequenze a livello di singola categoria farmacologica (ad esempio, gli antiemetici antagonisti corrispondono al $14 \%$ di tutti i trattamenti nel braccio A e al 9\% nel braccio B), anche per quanto riguarda $\mathrm{i}$ trattamenti concomitanti i due bracci appaiono complessivamente paragonabili: 4,8 trattamenti in media per paziente, che insieme comportano un costo di $€ 273$, nel braccio A; mentre nel braccio B si riportano per paziente 4,4 trattamenti e un costo di $€ 223$ (Tabella 9).

\subsection{Il costo delle trasfusioni}

Le emazie concentrate sono state praticamente l'unico emoderivato utilizzato nel NAVoTRIAL01 per le trasfusioni (13 nel braccio A e 6 nel braccio B); una sola trasfusione di piastrine, nel braccio B. Il costo medio per paziente risulta di $€ 63$ nel braccio A e €44 nell'altro (Tabella 10).

\subsection{Riepilogo}

La Tabella 11 riporta $\mathrm{i}$ dati finali delle diverse componenti di costo, espresse come costi medi per paziente. 
Tabella 9 Costo dei trattamenti concomitanti (curativi)

\begin{tabular}{|c|c|c|c|c|c|c|}
\hline \multirow[b]{2}{*}{ Trattamenti (per categoria farmacologica) } & \multirow[b]{2}{*}{ Principio attivo } & \multirow[b]{2}{*}{$\begin{array}{l}\text { Costo per } 1 \\
\text { trattamento } \\
(€)\end{array}$} & \multicolumn{2}{|c|}{$\begin{array}{l}\text { Braccio A } \\
\text { VNRo + CDDP } \rightarrow \text { VNRo } \\
n=100\end{array}$} & \multicolumn{2}{|c|}{$\begin{array}{l}\text { Braccio B } \\
\text { PEM + CDDP } \rightarrow \text { CDDP } \\
n=51\end{array}$} \\
\hline & & & $\begin{array}{l}\text { Frequenza } \\
\text { del } \\
\text { trattamento }\end{array}$ & $\begin{array}{l}\text { Costo } \\
\text { complessivo } \\
(€)\end{array}$ & $\begin{array}{l}\text { Frequenza } \\
\text { del } \\
\text { trattamento }\end{array}$ & $\begin{array}{l}\text { Costo } \\
\text { complessivo } \\
(€)\end{array}$ \\
\hline Antimicotici & Fluconazolo & 40,2 & 12 & 482 & 10 & 402 \\
\hline Altri antibiotici & Amoxicillina & 4,21 & 29 & 122 & 26 & 109 \\
\hline Antidiarroici & Loperamide & 0,56 & 13 & 7 & 5 & 3 \\
\hline Antiemetici 5HT3 antagonisti & Ondansetron & 114,8 & 64 & 7.347 & 21 & 2.411 \\
\hline Antiemetici non 5HT3 antagonisti & Metoclopramide & 1,75 & 72 & 126 & 32 & 56 \\
\hline Stimolanti l'eritropoiesi (ESA) & Eritropoietina & 1.022 & 12 & 12.264 & 7 & 7.154 \\
\hline Fattori di crescita (G-CSF) & Filgrastim & 491 & 13 & 6.383 & 2 & 982 \\
\hline Analgesici non oppioidi & Paracetamolo & 2,07 & 159 & 329 & 67 & 139 \\
\hline Analgesici oppioidi & Tramadolo & 2,32 & 104 & 241 & 54 & 125 \\
\hline Totale trattamenti concomitanti & & & 478 & 27.302 & 224 & 11.381 \\
\hline $\mathrm{N}^{\circ}$ pazienti & & & & 100 & & 51 \\
\hline Costo medio per paziente & & & & 273 & & 223 \\
\hline
\end{tabular}

Tabella 10 Costo delle trasfusioni

\begin{tabular}{|c|c|c|c|c|c|c|c|c|}
\hline \multirow[b]{2}{*}{ Emoderivati } & \multirow[b]{2}{*}{$\begin{array}{l}\text { Tariffa dell'e- } \\
\text { moderivato } \\
(€)\end{array}$} & \multirow[b]{2}{*}{$\begin{array}{l}\text { Tariffa } \\
\text { ambulatorio } \\
(€)\end{array}$} & \multicolumn{3}{|c|}{$\begin{array}{l}\text { Braccio A } \\
\text { VNRo + CDDP } \rightarrow \text { VNRo } \\
n=100\end{array}$} & \multicolumn{3}{|c|}{$\begin{array}{l}\text { Braccio B } \\
\text { PEM + CDDP } \rightarrow \text { CDDP } \\
n=51\end{array}$} \\
\hline & & & $\begin{array}{l}\mathrm{N}^{\circ} \text { di } \\
\text { unità } \\
\text { trasfuse }\end{array}$ & $\begin{array}{l}\mathrm{N}^{\circ} \mathrm{di} \\
\text { trasfusioni }\end{array}$ & $\begin{array}{l}\text { Costo } \\
\text { complessivo } \\
(€)\end{array}$ & $\begin{array}{l}\mathrm{N}^{\circ} \text { di } \\
\text { unità } \\
\text { trasfuse }\end{array}$ & $\begin{array}{l}\mathrm{N}^{\circ} \mathrm{di} \\
\text { trasfusioni }\end{array}$ & $\begin{array}{l}\text { Costo } \\
\text { complessivo } \\
(€)\end{array}$ \\
\hline Emazie & 153 & 25,8 & 39 & 13 & 6.302 & 12 & 5 & 1.965 \\
\hline Piastrine & 115 & 25,8 & 0 & 0 & 0 & 2 & 1 & 256 \\
\hline Totale & & & 39 & 13 & 6.302 & 14 & 6 & 2.221 \\
\hline $\mathrm{N}^{\circ}$ pazienti & & & & & 100 & & & 51 \\
\hline Costo medio per paziente & & & & & 63 & & & 44 \\
\hline
\end{tabular}

Tabella 11 Tabella riassuntiva del costo medio a paziente

\begin{tabular}{|c|c|c|c|c|c|}
\hline \multirow[b]{2}{*}{ Componenti di costo } & \multicolumn{2}{|c|}{$\begin{array}{l}\text { Braccio A } \\
\text { VNRo + CDDP } \rightarrow \text { VNRo }\end{array}$} & \multicolumn{2}{|c|}{$\begin{array}{l}\text { Braccio B } \\
\text { PEM + CDDP } \rightarrow \text { CDDP }\end{array}$} & \multirow{2}{*}{$\frac{\mathbf{A}-\mathbf{B}}{€ / \text { paziente }}$} \\
\hline & $€ /$ paziente & $\%$ & $€ /$ paziente & $\%$ & \\
\hline Farmaci antitumorali & 1.763 & 39,0 & 13.615 & 92,1 & -11.852 \\
\hline Setting di somministrazione & 1.801 & 39,9 & 344 & 2,3 & 1.457 \\
\hline Eventi avversi con ospedalizzazione & 611 & 13,5 & 567 & 3,8 & 44 \\
\hline Trattamenti concomitanti & 273 & 6,2 & 223 & 1,5 & 50 \\
\hline Trasfusioni & 63 & 1,4 & 44 & 0,3 & 19 \\
\hline Totale & 4.511 & 100,0 & 14.793 & 100,0 & -10.282 \\
\hline
\end{tabular}

Più del $90 \%$ del costo per paziente nel trattamento con Pemetrexed è dovuto al farmaco (€13.615); mentre quasi 1 ' $80 \%$ del costo nel trattamento con Vinorelbine orale è dovuto in parte al farmaco $(€ 1.763,39 \%)$ e in parte alle mo- dalità di somministrazione (€1.801, 39,9\%). Le altre componenti di costo (eventi avversi, trattamenti concomitanti, trasfusioni) sono - in valore assoluto-abbastanza simili tra i due bracci di trattamento. 
L'ultima colonna della Tabella 11 indica l'effetto, in termini di variazione dei costi, che si consegue se si sceglie la strategia del braccio A invece dell'altra. Ad esempio, così facendo il costo medio per paziente relativamente ai farmaci antitumorali andrebbe incontro a una cospicua riduzione ( $€$ 11.852). Nel complesso, scegliendo la strategia del braccio A il costo medio totale per paziente si ridurrebbe ( $€$ 10.282), dando luogo a un risparmio dello stesso importo per il SSN italiano.

\subsection{Analisi di sensibilità}

\subsubsection{Le dosi dei farmaci antitumorali}

La variabilità delle dosi somministrate ai pazienti nel NAVoTRIAL01 risulta di fatto molto contenuta. I valori medi stimati di tali dosi sono statisticamente compresi entro intervalli di confidenza (IC, al 95\%) ristretti. Se si considera, a titolo di esempio, il caso di Vinorelbine orale nel periodo d'induzione, l'IC della dose media (126,2 mg) va da $\pm 1,3 \%$ della stessa; nel caso di Pemetrexed (dose media di 859,5 $\mathrm{mg}$ ), l'analogo parametro non va oltre $\pm 2 \%$.

Di conseguenza, risulta pure contenuto l'impatto della variabilità dei dosaggi sul costo per paziente dei farmaci antitumorali. Anche ipotizzando, in via particolarmente conservativa, di attribuire simultaneamente alle dosi medie nel braccio A il valore superiore del rispettivo IC e a quelle del braccio $\mathrm{B}$ il valore inferiore si avrebbe:

- variazione del costo medio per paziente dei farmaci tumorali nel braccio $\mathrm{A}:+1,2 \%$

- variazione del costo medio per paziente dei farmaci tumorali nel braccio $\mathrm{B}:-1,2 \%$

- variazione del risparmio medio per paziente conseguito trattando con Vinorelbine orale: $-2,7 \%$.

\subsubsection{Il prezzo degli antitumorali}

Il costo per paziente dei farmaci è il fattore che maggiormente influisce sul saldo economico dei due antitumorali a confronto (Tabella 11). Partendo da questa considerazione, nel paragrafo precedente è stata svolta un'analisi di sensibilità su una delle due variabili da cui quel costo dipende direttamente: la quantità somministrata.

L'analisi presentata in questo paragrafo è rivolta all' altra variabile: il prezzo, sul quale sono state eseguite due simulazioni, con l'obiettivo formale di trovare un punto di pareggio fra i due trattamenti. Eccone i risultati:

(1) Il prezzo di Vinorelbine orale potrebbe crescere fino a sette volte il valore di base, prima che fosse raggiunta l'indifferenza economica tra l'impiego di quel farmaco e l'impiego di Pemetrexed
(2) Il prezzo di Pemetrexed dovrebbe ridursi a circa un quarto dell'attuale, prima che fosse raggiunta la stessa condizione tra l'impiego dello stesso farmaco e l'impiego di Vinorelbine orale.

\subsubsection{Un confronto interregionale}

L'analisi del caso base mostra come le voci di costo che principalmente influenzano la spesa per i due rispettivi trattamenti a carico del SSN siano relative ai farmaci antitumorali e alla loro somministrazione (setting di somministrazione). Nel loro insieme, esse possono anche variare da Regione a Regione in base alle differenti normative locali.

$\mathrm{Al}$ fine di valutare in quale misura la tariffazione utilizzata per l'analisi del caso base fosse riconducibile a quella delle varie realtà regionali, è stata condotta un'indagine presso 13 Regioni italiane [32, 36-46].

Il setting predominante per la somministrazione di chemioterapia infusionale o orale è risultato l'out-patient che prevede un accesso in day-hospital o in ambulatorio.

Le Tabelle 12 e 13 riportano il costo della chemioterapia per singola somministrazione nei due bracci di trattamento in base ai costi unitari dei farmaci (Tabella 1) e ai dosaggi medi riscontrati nello studio (Tabella 6). Collegando questi valori alle metodologie di rimborso delle diverse Regioni considerate, si ottiene la situazione riportata nelle Tabelle 14 e 15 .

Nelle Tabelle 14 e 15, i dati significativi per l'analisi di sensibilità sono quelli contenuti nell'ultima colonna (evidenziata in grassetto). Essi rappresentano il costo di una somministrazione (tariffa di rimborso dell' accesso all'ospedale più il costo del farmaco, qualora non già compreso nella tariffa) sostenuto dal Servizio Sanitario nella corrispondente Regione.

Tali costi possono essere confrontati con quello del caso base (evidenziato).

Come si osserva, la maggior parte delle Regioni adotta tariffe e tipologia di rimborso abbastanza simili a quelle utilizzate nel caso base per quanto riguarda il trattamento sia con Vinorelbine orale + Cisplatino che con Pemetrexed + Cisplatino.

Per quanto riguarda, in particolare, il trattamento con Vinorelbine orale + Cisplatino, pur considerando all'interno di un ciclo d'induzione un secondo accesso per la somministrazione di Vinorelbine orale il giorno 8 (che può essere peraltro evitato se la somministrazione è domiciliare), il costo complessivo per ciclo per il SSN italiano è circa un terzo di un corrispettivo ciclo con Pemetrexed. Stessa considerazione può essere fatta per il periodo di mantenimento.

Nel caso di Vinorelbine orale + Cisplatino, inoltre, per la maggior parte delle Regioni considerate, la tariffa applicata per l'accesso in prima giornata comprende il costo della chemioterapia il cui importo, in base al dosaggio medio 
Tabella 12 Costo dei farmaci antitumorali rapportato ad una somministrazione. Braccio A

Tabella 13 Costo dei farmaci antitumorali rapportato ad una somministrazione. Braccio B
Dose media $(\mathrm{mg}) \quad$ Prezzo/mg $(€)$

Costo/somministrazione $(€)$

\section{Induzione Giorno 1}

\begin{tabular}{lccc} 
VNRo & 126,2 & 1,56 & 196,9 \\
CDDP & 136,7 & 0,35 & 47,8 \\
Totale & & & $\mathbf{2 4 4 , 7}$ \\
Induzione Giorno 8 & 1,56 & \\
VNRo & 126,1 & & $\mathbf{1 9 6 , 7}$ \\
Mantenimento Giorno 1/8 & 134,6 & 1,56 & $\mathbf{2 1 0 , 0}$ \\
VNRo & 136 & \\
\hline
\end{tabular}

\begin{tabular}{llll}
\hline & Dose media $(\mathrm{mg})$ & Prezzo/mg $(€)$ & Costo/somministrazione $(€)$ \\
\hline \multicolumn{2}{l}{ Induzione Giorno 1} & & \\
PEM & 859,5 & 2,22 & 1908,1 \\
CDDP & 127,3 & 0,35 & 44,6 \\
Totale & & & $\mathbf{1 . 9 5 2 , 6}$ \\
Mantenimento Giorno 1 & & \\
PEM & 872,8 & 2,22 & $\mathbf{1 . 9 3 7 , 6}$ \\
\hline
\end{tabular}

Tabella 14 Costo complessivo della chemioterapia (accesso + farmaco) per il SSN, rapportato a una somministrazione. Braccio A

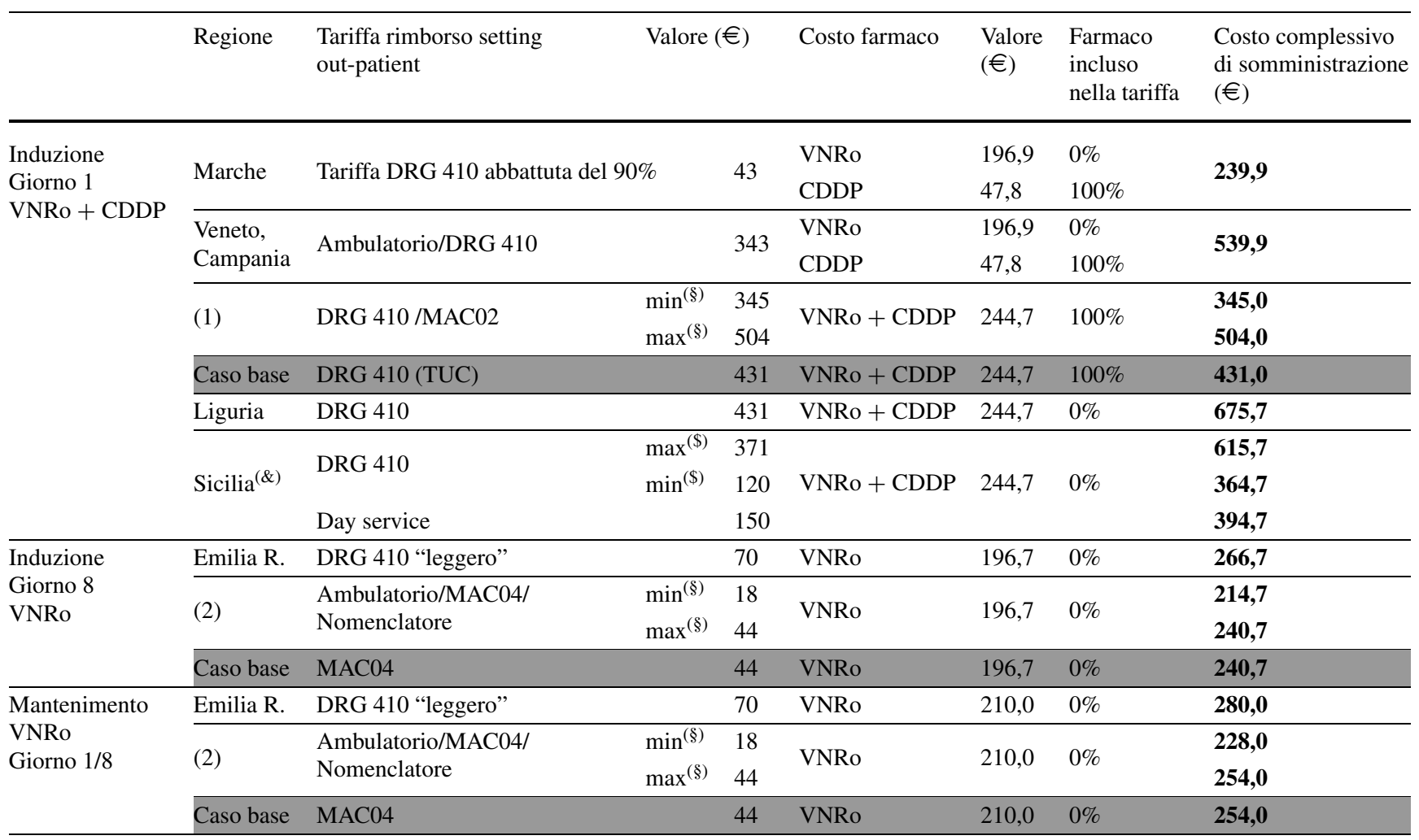

(1): Friuli VG, Emilia R, Lazio, Lombardia, Piemonte, Puglia, Sardegna, Toscana

(2): Campania, Friuli VG, Lazio, Liguria, Lombardia, Marche, Piemonte, Puglia, Sardegna, Sicilia, Toscana, Veneto

(§): $\mathrm{min} / \mathrm{max}$ interregionale

(\$): $\mathrm{min} / \mathrm{max}$ regionale

(\&): Le tariffe includono i costi di somministrazione del farmaco, gli esami ematochimici per la somministrazione del trattamento e la gestione degli effetti collaterali e gli esami radiologici di monitoraggio necessari durante tutto il periodo di trattamento 
Tabella 15 Costo complessivo della chemioterapia (accesso + farmaco) per il SSN, rapportato a una somministrazione. Braccio B

\begin{tabular}{|c|c|c|c|c|c|c|}
\hline Regione & $\begin{array}{l}\text { Tariffa rimborso setting } \\
\text { out-patient }\end{array}$ & Valore $(€)$ & Costo farmaco & Valore $(€)$ & $\begin{array}{l}\text { Farmaco } \\
\text { incluso } \\
\text { nella tariffa }\end{array}$ & $\begin{array}{l}\text { Costo complessivo } \\
\text { di somministrazione } \\
(€)\end{array}$ \\
\hline
\end{tabular}

\begin{tabular}{|c|c|c|c|c|c|c|c|c|}
\hline \multirow[t]{7}{*}{$\begin{array}{l}\text { Induzione } \\
\text { Giorno } 1 \\
\text { PEM + CDDP }\end{array}$} & (1) & $\begin{array}{l}\text { Tariffa DRG } 410 \text { abbattuta } \\
\text { del } 90 \%\end{array}$ & $\min ^{(\S)}$ & 38 & $\begin{array}{l}\text { PEM } \\
\text { CDDP } \\
\text { PEM } \\
\text { CDDP }\end{array}$ & $\begin{array}{l}1.908,1 \\
44,6 \\
1.908,1 \\
44,6\end{array}$ & $\begin{array}{l}0 \% \\
100 \% \\
0 \% \\
100 \%\end{array}$ & $\begin{array}{r}1.946,1 \\
1.951,1\end{array}$ \\
\hline & $\begin{array}{l}\text { Caso base, } \\
\text { Liguria, } \\
\text { Lombardia }\end{array}$ & MAC01 & & 44 & PEM + CDDP & $1.952,6$ & $0 \%$ & $1.996,6$ \\
\hline & Puglia, Toscana & DRG 410 & $\begin{array}{l}\min ^{(\S)} \\
\max ^{(\S)}\end{array}$ & $\begin{array}{l}371 \\
504\end{array}$ & $\mathrm{PEM}+\mathrm{CDDP}$ & $1.952,6$ & $100 \%$ & $\begin{array}{l}371,0 \\
504,0\end{array}$ \\
\hline & Campania & DRG 410 & & 345 & $\begin{array}{l}\text { PEM } \\
\text { CDDP }\end{array}$ & $\begin{array}{l}1.908,1 \\
44,6\end{array}$ & $\begin{array}{l}20 \% \\
100 \%\end{array}$ & $1.871,5$ \\
\hline & Emilia R & DRG 410 "pesante" & & 400 & $\begin{array}{l}\text { PEM } \\
\text { CDDP }\end{array}$ & $\begin{array}{l}1.908,1 \\
44,6\end{array}$ & $\begin{array}{l}50 \% \\
100 \%\end{array}$ & $1.354,1$ \\
\hline & Veneto & Ambulatorio $(\approx \mathrm{DH})$ & & 343 & $\begin{array}{l}\text { PEM } \\
\text { CDDP }\end{array}$ & $\begin{array}{l}1.908,1 \\
44,6\end{array}$ & $\begin{array}{l}0 \% \\
100 \%\end{array}$ & $2.251,1$ \\
\hline & $\operatorname{Sicilia}^{(\&)}$ & DRG 410 & $\begin{array}{l}\max ^{(\$)} \\
\min ^{(\$)}\end{array}$ & $\begin{array}{l}371 \\
120 \\
150\end{array}$ & PEM + CDDP & $1.952,6$ & $0 \%$ & $\begin{array}{l}2.323,6 \\
2.072,6 \\
2.102,6\end{array}$ \\
\hline \multirow[t]{8}{*}{$\begin{array}{l}\text { Mantenimento } \\
\text { PEM Giorno } 1\end{array}$} & (1) & $\begin{array}{l}\text { Tariffa DRG } 410 \text { abbattuta } \\
\text { del } 90 \%\end{array}$ & $\begin{array}{l}\min ^{(\S)} \\
\max ^{(\S)}\end{array}$ & $\begin{array}{l}38 \\
43\end{array}$ & PEM & $1.937,6$ & $0 \%$ & $\begin{array}{l}1.975,6 \\
1.980,6\end{array}$ \\
\hline & $\begin{array}{l}\text { Caso base, } \\
\text { Liguria, } \\
\text { Lombardia }\end{array}$ & MAC01 & & 44 & PEM & $1.937,6$ & $0 \%$ & $1.981,6$ \\
\hline & Puglia, Toscana & DRG 410 & $\begin{array}{l}\min ^{(\S)} \\
\max ^{(\S)}\end{array}$ & $\begin{array}{l}371 \\
504\end{array}$ & PEM & $1.937,6$ & $100 \%$ & $\begin{array}{l}371,0 \\
504,0\end{array}$ \\
\hline & Campania & DRG 410 & & 345 & PEM & $1.937,6$ & $20 \%$ & $1.895,1$ \\
\hline & Emilia R & DRG 410 "pesante" & & 400 & PEM & $1.937,6$ & $50 \%$ & $1.368,8$ \\
\hline & Veneto & Ambulatorio $(\approx \mathrm{DH})$ & & 343 & PEM & $1.937,6$ & $0 \%$ & $2.280,6$ \\
\hline & \multirow[t]{2}{*}{$\operatorname{Sicilia}^{(\&)}$} & DRG 410 & $\begin{array}{l}\max ^{(\$)} \\
\min ^{(\$)}\end{array}$ & $\begin{array}{l}371 \\
120\end{array}$ & PEM & $1.937,6$ & $0 \%$ & $\begin{array}{l}2.308,6 \\
2.057,6\end{array}$ \\
\hline & & Day service & & 150 & & & & $2.087,6$ \\
\hline
\end{tabular}

(1): Friuli VG, Lazio, Marche, Piemonte, Sardegna

(§): $\mathrm{min} / \mathrm{max}$ interregionale

(\$): $\mathrm{min} / \mathrm{max}$ regionale

(\&): Le tariffe includono i costi di somministrazione del farmaco, gli esami ematochimici per la somministrazione del trattamento e la gestione degli effetti collaterali e gli esami radiologici di monitoraggio necessari durante tutto il periodo di trattamento

utilizzato nel NAVoTRIAL01, risulta inferiore a quello della tariffa stessa, consentendo così un recupero parziale dei costi sostenuti per la somministrazione.

A corollario, è interessante segnalare come una recente indagine condotta in Italia dall' Associazione Italiana di Oncologia Medica [47] sull'utilizzo della chemioterapia orale, abbia mostrato come nella maggior parte dei casi $(75 \%)$ il costo del farmaco orale venga rimborsato separatamente. Questo è quanto è stato considerato nel nostro caso base (Vinorelbine orale nel giorno 8 e nel mantenimento) e quanto la presente analisi interregionale conferma.

\section{Discussione}

E' stata svolta un'analisi economica sui costi associati alla chemioterapia sulla base di quanto osservato nello studio internazionale di fase II NAVoTRIAL01 [25] in cui pazienti con NS-NSCLC sono stati randomizzati a ricevere 4 cicli di induzione di Vinorelbine orale + Cisplatino seguito da mantenimento con sola Vinorelbine orale o 4 cicli di induzione Pemetrexed + Cisplatino seguito da Pemetrexed come mantenimento. 
Entrambi gli approcci di trattamento, in base al disegno dello studio, sono risultati efficaci. Da esso sono stati derivati i dati di consumo delle risorse sanitarie.

Data l'importante mole di dati, si sono rese necessarie alcune semplificazioni quali quelle adottate per la stima del costo dei trattamenti concomitanti (cfr. Cap. 2.3.4). Sono ad esempio stati considerati i soli trattamenti prescritti con intento curativo in seguito all'insorgenza di eventi avversi principalmente correlabili al trattamento chemioterapico, ritenendosi le terapie di premedicazione standard ed equivalenti nei due bracci (non considerando la supplementazione con acido folico, corticosteroidi e vitamina B12 necessarie per Pemetrexed, la terapia antiemetica e l'ottava giornata dello schema con Vinorelbine orale).

In generale il peso economico di aspetti clinici quali ricoveri per SAE, trasfusioni e terapia concomitante, è risultato contenuto e confrontabile tra i due bracci (costo medio a paziente: $947 €$ nel braccio A e $834 €$ nel braccio B) pur essendo in alcuni casi la tipologia di evento differente (i.e. ricoveri per eventi non ematologici). Anche la frequenza con cui tali interventi si sono resi necessari era simile nei due bracci, tenendo in considerazione il rapporto 2:1 della randomizzazione.

Un limite dell'analisi potrebbe essere un grado di controvertibilità delle ipotesi con cui sono stati adottati i regimi tariffari relativamente ai setting di somministrazione, la cui scelta, oltretutto, finisce per avere una ricaduta anche sul costo dei farmaci antitumorali da attribuire al SSN.

D'altra parte, con l'analisi di sensibilità (confronto interregionale al Par. 3.7.3.) si è potuto valutare la limitata influenza di tali ipotesi sul risultato finale.

A questo proposito, e più in generale, l'analisi di sensibilità ha messo in evidenza la piena tenuta dei risultati di base anche a fronte di ipotesi particolarmente conservative. Questo può derivare dal fatto che il margine di risparmio per paziente trattato con Vinorelbine orale è in partenza più del doppio del rispettivo costo complessivo di trattamento, ed è quindi in grado di rimanere economicamente apprezzabile pur nel cambiamento degli scenari.

L'analisi interregionale, riportando il costo per somministrazione in base ai dosaggi medi dei due trattamenti riscontrati nello studio NAVoTRIAL01 nelle rispettive fasi d'induzione e mantenimento, mostra anche come il trattamento con Vinorelbine orale e Cisplatino possa comportare, per gli Enti, un "recupero" di risorse economiche.

Attualmente non sono disponibili in letteratura pubblicazioni che permettano di avere qualche riscontro diretto con gli outcomes economici (costi e risparmi) della presente analisi.

In un setting di malattia più precoce, una valutazione economica retrospettiva [48] è stata condotta (con l'analisi di costo efficacia, nella prospettiva del servizio sanitario canadese) sui risultati dello studio clinico National Cancer Istitute of Canada Clinical Trials Group (NCIC CTG)
JBR.10, conclusosi nel 2004 [49]. Tale studio metteva a confronto Vinorelbine + Cisplatino versus altre terapie standard nel trattamento di pazienti con carcinoma polmonare non a piccole cellule in fase iniziale (IB-II) dopo resezione, concludendo — sulla base dei risultati molto favorevoli-che per tali pazienti questa chemioterapia adiuvante di terza generazione veniva ormai a porsi come il nuovo standard di cura.

I costi presi in considerazione nella suddetta valutazione economica (focalizzata sul sottoinsieme costituito dai 172 pazienti canadesi partecipanti al NCIC CTG JBR.10) erano principalmente quelli per chemioterapia, ricoveri (ambulatoriali e in degenza), interventi chirurgici e radiologici, trattamenti concomitanti, trasfusioni. Nel braccio di Vinorelbine, il costo medio complessivo per paziente è risultato pari a 40.403 dollari canadesi (\$) che, rapportati a una sopravvivenza media di 7 anni (dati, entrambi, non scontati), corrispondono a un valore medio annuo di circa $\$ 5.800$ che corrisponde a poco meno di $€ 4.500$.

Da questa analisi si evince che il costo complessivo medio per paziente nel braccio A (€4.511) rapportato alla sopravvivenza complessiva mediana nello stesso braccio $(10,2$ mesi) porta ad un valore annuo di $€ 5.307$ che appare paragonabile a quello riportato appena sopra considerando gli elementi di eterogeneità fra i due studi di origine.

In un'analisi di minimizzazione dei costi [50] condotta nel Regno Unito che ha confrontato la formulazione orale di Vinorelbine con altre terapie per via endovenosa (Vinorelbine, Gemcitabina, Paclitaxel, Docetaxel) utilizzate in monochemioterapia per il trattamento della prima linea del NSCLC, si è dimostrato come la formulazione orale fosse l'opzione terapeutica che comportava il maggior risparmio di risorse dal punto di vista del Sistema Sanitario Inglese anche se questo confronto non tiene conto dell' attuale utilizzo di farmaci generici a minor costo.

Per quanto riguarda l'altro agente chemioterapico considerato in questo studio, da un'analisi di minimizzazione dei costi svolta nella prospettiva economica dell'ospedale con riferimento alla pratica clinica italiana, il costo/paziente complessivo (farmaco, somministrazione, premedicazione, monitoraggio e supporto, eventi avversi) per un mese di terapia con Pemetrexed in monochemioterapia nella seconda linea del NSCLC, risultava pari a $€ 3.324$ ( $€ 1.669$ con Erlotinib e $€ 2.569$ con Docetaxel) [51]. Questo risultato, pur tenendo in considerazione gli elementi di eterogeneità tra le due analisi, risulta essere dello stesso ordine di grandezza di quanto riportato nella presente analisi (Par. 3.7.3).

\section{Conclusioni}

Questa analisi dimostra che un paziente con NS-NSCLC trattato con Pemetrexed + Cisplatino e mantenimento alle 
condizioni del NAVoTRIAL01 comporta per il SSN italiano un costo medio circa 3 volte superiore di un paziente trattato con Vinorelbine orale + Cisplatino seguito da mantenimento alle medesime condizioni. In base al disegno dello studio entrambi i regimi si sono mostrati efficaci nel controllo della malattia e in termini di obiettivi secondari (sopravvivenza globale, sopravvivenza libera da progressione). Il trattamento con Vinorelbine orale appare dunque un'alternativa economicamente conveniente per il SSN italiano, dal momento che consente un risparmio di costi dell'ordine di $€ 10.000$ in media per paziente. Tali conclusioni (per il momento supportate da un'ampia analisi di sensibilità) andrebbero confermate in uno studio clinico di fase III.

Ringraziamenti Lo studio è stato condotto grazie a un supporto incondizionato di Pierre Fabre Pharma S.r.l. Centri Italiani che hanno partecipato al NAVoTRIAL01 (Principal Investigator): Oncologia Medica 2 Istituto Oncologico Veneto IRCCS, Padova (A. Favaretto); Oncologia Medica 1 AO Città della Salute e della Scienza, Torino (L. Ciuffreda); Oncologia Medica AUSL Rimini Ospedale Cervesi, Cattolica (M. Nicolini); UOC Oncologia Medica Azienda USL12Viareggio, Ospedale Versilia, Lido di Camaiore (D. Amoroso); Oncologia Medica A.O.U. Policlinico S. Orsola Malpighi, Bologna (B. Melotti); Clinica di Oncologia Medica A.O.U. Ospedali Riuniti, Ancona (S. Cascinu); SS Oncologia ASL3-Genovese Ospedale Villa Scassi, Genova (M. Mencoboni).

Gli autori dichiarano di non avere conflitti di interesse relativi al contenuto del presente articolo.

Open Access This article is distributed under the terms of the Creative Commons Attribution Noncommercial License which permits any noncommercial use, distribution, and reproduction in any medium, provided the original author(s) and the source are credited.

\section{Bibliografia}

1. I numeri del cancro in Italia 2013. Rapporto AIOM-AIRTUM. www.registri-tumori.it.

2. Shepherd F, et al. ASCO 2013 Educational book

3. Peters S, et al (the ESMO Guidelines Working Group). Metastatic non-small-cell lung cancer (NSCLC): ESMO clinical practice guidelines for diagnosis, treatment and follow-up. Ann Oncol. 2012;23:7.

4. Linee Guida Neoplasie del Polmone, AIOM 2013

5. Scagliotti GV, et al. Phase III study comparing cisplatin plus gemcitabine with cisplatin plus Pemetrexed in chemotherapy-naïve patients with advanced-stage non-small-cell lung cancer. J Clin Oncol. 2008;26(21).

6. Rodrigues-Pereira J, et al. A randomized phase III trial comparing pemetrexed/carboplatin and docetaxel/carboplatin as first-line treatment for advanced, non-squamous non-small cell lung cancer. J Thorac Oncol. 2011;6(11).

7. Patel JD, et al. PointBreak: a randomized phase III study of pemetrexed plus carboplatin and bevacizumab followed by maintenance pemetrexed and bevacizumab versus paclitaxel plus carboplatin and bevacizumab followed by maintenance bevacizumab in patients with stage IIIB or IV nonsquamous non-small-cell lung cancer. J Clin Oncol. 2013;31(34).

8. Scagliotti GV, et al. Phase III randomized trial comparing three platinum-based doublets in advanced non-small-cell lung cancer. Italian Lung Cancer Project. J Clin Oncol. 2002;20(21).
9. Le Chevalier T, et al. Long term analysis of survival in the European randomized trial comparing Vinorelbine/cisplatin to vindesine/cisplatin and Vinorelbine alone in advanced non-small cell lung cancer. The Oncologist. 2001;6(Suppl 1):8-11.

10. Wozniak AJ, et al. Randomized trial comparing cisplatin with cisplatin plus Vinorelbine in the treatment of advanced non-smallcell lung cancer: a Southwest Oncology Group study. J Clin Oncol. $1998 ; 16(7)$.

11. Kelly K, et al. Randomized phase III trial of paclitaxel plus carboplatin versus Vinorelbine plus cisplatin in the treatment of patients with advanced non-small-cell lung cancer: a Southwest Oncology Group trial. J Clin Oncol. 2001;19(13).

12. Jassem $\mathrm{J}$, et al. Oral Vinorelbine in combination with cisplatin: a novel active regimen in advanced non-small cell lung cancer (NSCLC). Ann Oncol. 2003;14:1634-9.

13. Jensen L, et al. Randomized cross-over study of patient preference for oral or intravenous Vinorelbine in combination with carboplatin in the treatment of advanced NSCLC. Lung Cancer 2008;62(1).

14. De Lena M, et al. Phase II trial of oral Vinorelbine in combination with Cisplatin followed by consolidation therapy with oral Vinorelbine in advanced NSCLC. Lung Cancer. 2005;48.

15. Tan EH, et al. Global Lung Oncology Branch trial 3 (GLOB3): final results of a randomised multinational phase III study alternating oral and i.v. Vinorelbine plus cisplatin versus docetaxel plus cisplatin as first-line treatment of advanced non-small-cell lung cancer. Ann Oncol. 2009;20.

16. Douillard JY, et al. Adjuvant Vinorelbine plus cisplatin versus observation in patients with completely resected stage IB-IIIA nonsmall-cell lung cancer (Adjuvant Navelbine International Trialist Association [ANITA]): a randomised controlled trial. Lancet Oncol. 2006;7.

17. Kolek V, et al. Oral Vinorelbine in combination with cisplatin or carboplatin in adjuvant chemotherapy of non-small cell lung cancer: a prospective multicentre study of tolerability and survival. In: 2013 world lung cancer conference P1.12-009; 2013.

18. James RD, et al. Improving chemotherapy capacity by switching from IV to oral Vinorelbine. Eur J Oncol Pharm. 2010;4.

19. Pirker R, et al. Prognostic factors in patients with advanced nonsmall cell lung cancer: data from the phase III FLEX study. Lung Cancer. 2012;77(2).

20. Fidias $P$, et al. Strategies for prolonged therapy in patients with advanced non-small-cell lung cancer. J Clin Oncol. 2010;28.

21. Brodowicz T, et al. Cisplatin and Gemcitabine first-line chemotherapy followed by maintenance Gemcitabine or best supportive care in advanced non-small cell lung cancer: a phase III trial. Lung Cancer. 2006;52.

22. Paz-Ares LG, et al. Paramount: final overall survival results of the phase III study of maintenance pemetrexed versus placebo immediately after induction treatment with pemetrexed plus cisplatin for advanced nonsquamous non-small-cell lung cancer. J Clin Oncol. 2013;13(23).

23. Ciuleanu T, et al. Maintenance pemetrexed plus best supportive care versus placebo plus best supportive care for non-small-cell lung cancer: a randomised double-blind, phase 3 study. Lancet. 2009;374.

24. Cappuzzo F, et al. Erlotinib as maintenance treatment in advanced non-small-cell lung cancer: a multicentre, randomized, placebo controlled phase 3 study. Lancet Oncol. 2010;11.

25. Bennouna $\mathrm{J}$, et al Prospective randomised phase II trial of oral Vinorelbine and cisplatin $(\mathrm{P})$ or Pemetrexed and $\mathrm{P}$ in first-line metastatic or locally advanced non-small cell lung cancer (M or LA NSCLC) with nonsquamous (Non SCC) histological type. NAVoTRIAL01: final results. J Clin Oncol. 2013;31(Suppl):8043. ASCO 2013. 
26. Eisenhauer EA, et al. New response evaluation criteria in solid tumours: revised RECIST guideline (version 1.1). Eur J Cancer. 2009;45.

27. Drummond MF, et al. Methods for the economic evaluation of health care programmes. 3rd ed. London: Oxford University Press; 2005; p. 379.

28. L'Informatore Farmaceutico. http://www.codifa.it (accesso del 15/04/2013).

29. Conferenza delle regioni e delle province autonome. Accordo interregionale per la compensazione della mobilità sanitaria. Versione in vigore per le attività dell'anno 2012. Allegato B-Farmaci oncologici ad elevato costo.

30. Ministero della salute. Remunerazione delle prestazioni di assistenza ospedaliera per acuti, assistenza ospedaliera di riabilitazione e di lunga degenza post acuzie e di assistenza specialistica ambulatoriale. Decreto 18 ottobre 2012. Art. 2-Aggiornamento delle tariffe per le prestazioni di assistenza ospedaliera.

31. Conferenza delle regioni e delle province autonome. Accordo interregionale per la compensazione della mobilità sanitaria. Versione in vigore per le attività dell' anno 2012. Tariffa Unica Convenzionale (TUC) per le prestazioni di assistenza ospedaliera per acuti.

32. Regione Lombardia-Deliberazione $N^{\circ}$ IX/2946 del 25/01/2012. Allegato 4-Tariffe pacchetti di Macroattività Ambulatoriale Complessa e ad alta integrazione di risorse (MAC).

33. Conferenza delle regioni e delle province autonome. Accordo interregionale per la compensazione della mobilità sanitaria. Versione in vigore per le attività dell'anno 2012. Farmaci a somministrazione diretta (Flusso F).

34. Conferenza delle regioni e delle province autonome. Accordo interregionale per la compensazione della mobilità sanitaria. Versione in vigore per le attività dell'anno 2012. Allegato $\mathrm{C}$ - prodotti (sangue ed emocomponenti) previsti da D.M. 5 novembre $1996 \mathrm{e}$ dall' accordo stato-regioni del 24 luglio 2003.

35. Ministero della salute. Remunerazione delle prestazioni di assistenza ospedaliera per acuti, assistenza ospedaliera di riabilitazione e di lunga degenza post acuzie e di assistenza specialistica ambulatoriale. Decreto 18 ottobre 2012, G.U. Suppl. n. 8, 28 gennaio 2013. Allegato 3-Prestazioni di assistenza specialistica ambulatoriale.
36. Regione Puglia Deliberazione della Giunta Regionale n. 951 DEL 13-05-2013.

37. Regione Marche Delibera n. 561 del 15/3/2010.

38. Regione Autonoma della Sardegna Deliberazione n. 26/35 DEL 6.7.2010.

39. Regione Emilia Romagna Determinazione 525/2013.

40. Regione Campania Delibera n. 1034, 2006.

41. Regione Lazio Decreto n. 50, 14 giugno 2010.

42. Regione Sicilia D.A. n. 954, 2013.

43. Regione Veneto Decreto n. 47, 2013, Delibera Giunta Regionale n. 95, 31 gennaio 2012.

44. Regione Toscana Suppl. Boll. Uff. n. 3 del 19.1.2005; D.G.R. 1184/2008; www.regione.toscana.it: nomenclatore tariffario regionale.

45. Regione Piemonte Delibera Giunta Regionale 29/3/2010 n. 97-13750 (Boll. Uff. n. 17 del 29/4/2010).

46. Centro Studi Assobiomedica-Analisi n. 16, dicembre 2012. http://www.assobiomedica.it/static/upload/ana/analisi_16.pdf.

47. Gori S, et al. Impact of the use of oral anticancer drugs on activity of Italian oncology practices: results of a survey conducted by the Italian Society of Medical Oncology (AIOM). Tumori. 2013;99.

48. $\mathrm{Ng} \mathrm{R}$, et al. Economic analysis of NCIC CTG JBR.10: a randomized trial of adjuvant Vinorelbine plus cisplatin compared with observation in early stage in non-small-cell lung cancer-a report of the Working Group on Economic Analysis, and the Lung Disease Site Group, National Cancer Istitute of Canada Clinical Trials Group. J Clin Oncol. 2007;25(16).

49. Randomized phase III trial of Vinorelbine plus cisplatin compared with observation in completely resected stage IB and II nonsmall-cell lung cancer: updated survival analysis of JBR-10. J Clin Oncol. 2010;28(1)

50. Le Lay $\mathrm{K}$, et al. Comparative cost-minimisation of oral and intravenous chemotherapy for first-line treatment of non-small cell lung cancer in the UK NHS system. Eur J Health Econ. 2007;8(2).

51. Capri S, et al. Valutazione economica di erlotinib, docetaxel e Pemetrexed nel trattamento di seconda linea del carcinoma polmonare non a piccole cellule. PharmacoEcon, Ital Res Artic. 2007;9(2). 